\title{
ASYMPTOTIC SOLUTION FOR LARGE PRANDTL NUMBER FOR THE FLOW OF A POWER-LAW FLUID THROUGH A POROUS MEDIUM OVER A ROTATING DISK WITH HEAT TRANSFER AND VISCOUS DISSIPATION
}

\author{
Hazem Ali Attia ${ }^{1}$, Karem Mahmoud Ewis ${ }^{1}$ and Mostafa A. M. Abdeen ${ }^{2}$ \\ ${ }^{1}$ Department of Engineering Mathematics and Physics, Faculty of Engineering, \\ Fayoum University, El-Fayoum-63514, Egypt \\ E-mail: ah1113@yahoo.com \\ ${ }^{2}$ Department of Engineering Mathematics and Physics, Faculty of Engineering, \\ Cairo University, Giza 12211, Egypt \\ E-mail: mostafa_a_m_abdeen@hotmail.com
}

(Received September 6, 2014)

\begin{abstract}
The steady flow with heat transfer through a porous medium of a nonNewtonian power-law fluid due to the uniform rotation of a disk of infinite extent is studied. The porous medium is assumed to obey Darcy's model which accounts for the drag exerted linearly by the porous medium on the steady flow. Von Karman similarity transformation is used to transform the governing boundary layer partial differential equations to ordinary differential equations. Therefore, the resulting momentum equations as well as the energy equations including the viscous dissipation term are solved asymptotically for large values of the porosity parameter and Prandtl number.
\end{abstract}

Keywords: Rotating disk, porous medium, non-Newtonian fluid, power-law fluid, heat transfer, viscous dissipation, asymptotic solution.

\section{INTRODUCTION}

Von Karman rotating disk problem [1] is one of the classical problems in fluid mechanics that was introduced by VON KARMAN 1921 in the steady state. A similarity transformation was used to reduce the governing partial differential equations to ordinary differential equations. COCHRAN [2] obtained asymptotic solution for the reduced system of ordinary differential equations. The extension of von Karman problem to the case of flow of non-Newtonian power-law electrically conducting fluids under the action of an external uniform magnetic field was introduced by ANDERSON [3]. MILlSAPS and POHLHAUSEN [4] and SPARROW and GREGG [5] studied the heat transfer from the surface of a rotating disk maintained at a constant temperature in the steady state case for various values of Prandtl numbers. The extensions of the heat transfer problems studied in $[4,5]$ to the transient state was done by ATTIA [6] in the presence of uniform magnetic field. 
In the present paper, the steady flow through a porous medium due to the rotation of an infinite rotating infinite disk of a non-Newtonian power-law fluid with heat transfer is investigated. The flow in the porous medium is assumed to be based on the Darcy's law which accounts for the drag exerted by the porous medium [7-8]. A similarity transformation is used to transform the governing boundary layer nonlinear partial differential equations to nonlinear ordinary differential equations. An asymptotic solution for the resulting nonlinear governing momentum and energy equations including the viscous dissipation is obtained for large values of the porosity parameter and Prandtl number.

\section{THE GOVERNING EQUATIONS}

In this paper we consider the non-Newtonian fluid that obeys the Ostwald-de Waele power-law model given by [3]

$\vec{\tau}=2 \mu \vec{D}=2 K\left(2 \vec{D}_{i j} \vec{D}_{i j}\right)^{(n-1) / 2} \vec{D}$

where $\vec{\tau}$ is the sheer stress tensor, $\vec{D}$ is the rate-of-strain tensor, $\mu$ is the viscosity of fluid, and $K$ and $n$ are the consistency coefficient and the power-law index, respectively.

The flow of the fluid is assumed to be in the half-space $z>0$ above the infinite disk, and is governed by the following boundary layer equations for conservation of mass and momentum,

$\frac{\partial u}{\partial r}+\frac{u}{r}+\frac{\partial w}{\partial z}=0$

$\rho\left(u \frac{\partial u}{\partial r}-\frac{v^{2}}{r}+w \frac{\partial u}{\partial z}\right)=-\frac{\partial p}{\partial r}+\frac{\partial}{\partial z}\left(\mu \frac{\partial u}{\partial z}\right)-\frac{K}{k} u$

$\rho\left(u \frac{\partial v}{\partial r}+\frac{u v}{r}+w \frac{\partial v}{\partial z}\right)=\frac{\partial}{\partial z}\left(\mu \frac{\partial v}{\partial z}\right)-\frac{K}{k} v$,

$0=-\frac{\partial p}{\partial z}-\frac{K}{k} w$

where $(r, \varphi, z)$ are the cylindrical coordinates, $u, v$, and $w$ are the components in the radial, azimuthal and axial directions, respectively, $p$ is the pressure which is assumed to be a function of $z$ only and $\mu$ is the viscosity function given as

$\mu=K\left(\left(\frac{\partial u}{\partial z}\right)^{2}+\left(\frac{\partial v}{\partial z}\right)^{2}\right)^{(n-1) / 2}$

which simplifies to $\mu=K$ for the particular parameter value $n=1$ (for Newtonian fluids) and $k$ is the Darcy permeability [7-8]. The last term in the right-side of each of Eqs. (3), (4) and (5) represents the Darcy force exerted by the fibers of the porous medium [7-8]. The boundary conditions for the flow problem are given by

$z=0: u=w=0, v=\omega r$
$z \rightarrow \infty: u \rightarrow 0, v \rightarrow 0$

Equation (5) can be used to determine the pressure across the boundary layer when the axial velocity component is known. Introducing the generalized dimensionless similarity variable $\eta[3]:$

$\eta=z\left(\frac{\omega^{2-n}}{K / \rho}\right)^{1 /(n+1)} \cdot r^{(1-n) /(1+n)}$

together with the known von Karman similarity variables

$u=r \omega F(\eta), v=r \omega G(\eta), w=\left(\frac{\omega^{1-2 n}}{K / \rho}\right)^{-1 /(n+1)} \cdot r^{(n-1) /(n+1)} H(\eta)$. 
the boundary layer Eqs. (2)-(4) transform into the ordinary differential equations [3]

$$
\begin{aligned}
& H^{\prime}=-2 F-\frac{1-n}{n+1} \eta F^{\prime}, \\
& F^{2}-G^{2}+\left(H+\frac{1-n}{n+1} F \eta\right) F^{\prime}+m F=\left(\left(F^{\prime 2}+G^{\prime 2}\right)^{(n-1) / 2} F^{\prime}\right)^{\prime}, \\
& 2 F G+\left(H+\frac{1-n}{n+1} F \eta\right) G^{\prime}+m G=\left(\left(F^{\prime 2}+G^{\prime 2}\right)^{(n-1) / 2} G^{\prime}\right)^{\prime},
\end{aligned}
$$

where $m$ is the porosity parameter and the primes denote differentiation with respect to the similarity variable $\eta$

$m=\frac{K}{k \rho \omega}$.

The boundary conditions (7) and (8) transform to

$\eta=0: F=0, G=1, H=0$

$\eta \rightarrow \infty: F \rightarrow 0, G \rightarrow 0$,

\section{Asymptotic solution for the flow equations for large $m$}

Following the procedures given in [3] it is known that the Darcy body force tends to reduce the radial $(F)$ and azimuthal $(G)$ velocity components which, consequently, reduces the axial velocity $(H)$. For high values of the porosity parameter $m$, both $F$ and $H$ may vanish, whereas $G$, close to the surface of the disk, remains finite due to the no-slip condition given in Eq. (1). In the limit $m \rightarrow \infty$, the azimuthal momentum Eq. (13) reduces to [3] $\left(\left(G^{\prime 2}\right)^{(n-1) / 2} G^{\prime}\right)^{\prime}=m G$.

Let us furthermore assume that $G^{\prime} \leq 0$. Then, with $G^{\prime \prime} d G=G^{\prime} d G^{\prime}$, this becomes [3]

$-n\left(-G^{\prime}\right)^{n} d G^{\prime}=m G d G$

which by integration gives

$-G^{\prime}=\left(\frac{n+1}{2 n} m G^{2}\right)^{1 / n+1}$

where it is assumed that both $G$ and $G^{\prime}$ tend to zero sufficiently far away from the disk. Integrated Eq. (19) to get the closed-form solution [3]

$G(\eta)=\exp (-\sqrt{m} \eta)$ for $n=1$,

$G(\eta)=(1+A \eta)^{B}$ for $n \neq 1$,

where $A=\frac{1-n}{1+n}\left(\frac{1+n}{2 n} m\right)^{1 /(1+n)}, B=-\frac{(1+n)}{1-n}$

Using Eq. (20) we find that

$G^{\prime}(\eta)=-\sqrt{m} \exp (-\sqrt{m} \eta)$ for $n=1$,

$G^{\prime}(\eta)=A B(1+A \eta)^{B-1}$ for $n \neq 1$,

Also the wall-gradient can be estimated as [3]

$-G^{\prime}(0)=\left(\frac{n+1}{2 n} m\right)^{1 /(n+1)}$

which gives the torque required to maintain the rotation of the disk with constant angular velocity [3].

The energy equation including the viscous dissipation takes the form [4-5]; 
$\rho c_{p}\left(u \frac{\partial T}{\partial r}+w \frac{\partial T}{\partial z}\right)=k \frac{\partial^{2} T}{\partial z^{2}}+\frac{k}{r} \frac{\partial}{\partial r}\left(\frac{1}{r} \frac{\partial T}{\partial r}\right)+\mu\left\{\left(\frac{\partial u}{\partial z}\right)^{2}+\left(\frac{\partial v}{\partial z}\right)^{2}\right\}$

where $T$ is the temperature of the fluid, $c_{p}$ is the specific heat at constant pressure of the fluid, and $k$ is the thermal conductivity of the fluid. The boundary conditions for the energy problem are that, by continuity considerations, the temperature equals $T_{w}$ at the surface of the disk. Far from the surface of the disk, $T$ tends to $T_{\infty}$ where $T_{\infty}$ is the temperature of the ambient fluid. In terms of the non-dimensional variable

$\theta(\zeta)=\left(T-T_{\infty}\right) /\left(T_{w}-T_{\infty}\right)$

and using von Karman transformations, Eq. (23) takes the form;

$\theta^{\prime \prime}-\operatorname{Pr} H \theta^{\prime}+\operatorname{Pr} E c\left(\left(F^{\prime}\right)^{2}+\left(G^{\prime}\right)^{2}\right)^{(n+1) / 2}=0$

where

$\operatorname{Pr}=\mu_{0}^{2 /(n+1)} c_{p}\left(\omega^{3} r^{2}\right)^{\frac{n-1}{n+1}} / k$ is the Prandtl number,

$E c=\omega^{2} r^{2} / c_{p}\left(T_{w}-T_{\infty}\right)$ is the Eckert number.

The boundary conditions for the temperature problem are expressed in terms of $\theta$ as $\theta(0)=1, \theta \rightarrow 0$ as $\zeta \rightarrow \infty$

\section{Asymptotic solution for the energy equation for large $\boldsymbol{m}$ and large $\mathrm{Pr}$}

For high values of the porosity parameter $m, F$ may vanish, and accordingly $F^{\prime}$ vanishes. For very large values of Prandtl number Pr, the thermal boundary later is confined to a smaller and smaller portion of the velocity boundary layer. This guides us to write $H$ in terms of a series expansion about $\eta=0$ [4,5]. Therefore, we obtain

$H=H(0)+H^{\prime}(0) \eta+\frac{H^{\prime \prime}(0)}{2} \eta^{2}+\ldots$

Using the conditions, $H(0)=H^{\prime}(0)=0$, we get

$H=H^{\prime \prime}(0) \frac{\eta^{2}}{2}$

For small values of $\eta$. Substituting for $H$ from Eq. (27) into Eq. (24) we end up with a second order inhomogeneous linear variable coefficients ordinary differential equation which can be solved under the boundary conditions given by Eq. (25) and whose exact solution is given by

$\theta(\eta)=\left(u_{o}+\int_{0}^{\eta} \frac{g(\eta) h(\eta)}{g^{\prime}(\eta)} d \eta\right)+\left(v_{o}-\int_{0}^{\eta} \frac{h(\eta)}{g^{\prime}(\eta)} d \eta\right) g(\eta)$

where

$u_{o}=\frac{\left(\int_{0}^{\infty} \frac{g(\eta) h(\eta)}{g^{\prime}(\eta)} d \eta\right)-\left(1+\int_{0}^{\infty} \frac{h(\eta)}{g^{\prime}(\eta)} d \eta\right) g(\infty)}{1+g(\infty)}$ 
$v_{o}=\frac{\left(1-\int_{0}^{\infty} \frac{g(\eta) h(\eta)}{g^{\prime}(\eta)} d \eta\right)+\left(2+\int_{0}^{\infty} \frac{h(\eta)}{g^{\prime}(\eta)} d \eta\right) g(\infty)}{1+g(\infty)}$

$h(\eta)=A^{2} B^{2} \operatorname{Pr} E c(1+A \eta)^{2(B-1)}$

$g(\eta)=1-\frac{\int_{0}^{\left(-\operatorname{Pr} H^{\prime \prime}(0) / 6\right)^{1 / 2}} \eta \exp \left(-\xi^{3}\right) d \xi}{\int_{0}^{\infty} \exp \left(-\xi^{3}\right) d \xi}$

$g(\infty)=1-\frac{\int_{0}^{\left(-\operatorname{Pr} H^{\prime \prime}(0) / 6\right)^{1 / 2}} \eta_{\infty} \exp \left(-\xi^{3}\right) d \xi}{\int_{0}^{\infty} \exp \left(-\xi^{3}\right) d \xi}$

\section{CONCLUSIONS}

The steady flow with heat transfer through a porous medium of a power-law fluid above an infinite rotating disk was studied in the case of large values of due to the rotation of an infinite disk through a porous medium was studied in the case of large values of porosity parameter and Prandtl number. Von Karman similarity transformations were used to transformed the set of governing partial differential equations to ordinary differential equations and then, an asymptotic solution for the resulting system of governing nonlinear ordinary differential equations was obtained for large porosity parameter and large Prandtl number.

\section{References:}

[1] VON KARMAN, T., Uber laminare und turbulente reibung, ZAMM 1 (4) (1921) 233-235.

[2] Cochran, W.G., The flow due to a rotating disk, Proc. Cambridge Philos. Soc. 30 (3) (1934) 365-375.

[3] Andersson, H.I., DE KoRTE, E., MHD flow of a power-law fluid over a rotating disk, European Journal of Mechanics B/Fluids 21 (2002) 317-324.

[4] Millsaps, K. and PohlHausen, K., Heat transfer by laminar flow from a rotating disk, J. of the Aeronautical Sciences 19 (1952) 120-126.

[5] Sparrow, E.M. and GREGG, J.L., Mass transfer, flow, and heat transfer about a rotating disk, J. of Heat Transfer, ASME 82 (4) (Nov 1960) 294-302.

[6] AtTIA, H.A., Unsteady MHD flow near a rotating porous disk with uniform suction or injection, Fluid Dynamics Research 23 (1998) 283-290. 
[7] Ingham, D.B. and PoP, I., Transport phenomena in porous media, Pergamon, Oxford, 2002.

[8] Khaled, A.R.A. and VAfai, K., The role of porous media in modeling flow and heat transfer in biological tissues, Int. J. Heat Mass Transf. 46 (2003) 4989-5003. 